SCIENTIFIC REPORT

\title{
Risk factors of acute endophthalmitis after cataract extraction: a case-control study in Asian eyes
}

\author{
T Y Wong, S-P Chee
}

Br J Ophthalmol 2004;88:29-31

\begin{abstract}
Aim: To describe risk factors of acute endophthalmitis after cataract extraction in an Asian population

Methods: A retrospective, case-control study. Cases $(n=34)$ were patients with acute endophthalmitis presenting within 6 weeks after cataract surgery. Three controls per case $(n=102)$ were randomly selected from the cataract surgery list matched on the date of operation of cases.

Results: Few risk factors were identified. In multivariable analysis, endophthalmitis was associated with silicone intraocular lens lodds ratio 5.1, 95\% confidence intervals, 1.2 to 21.6 , compared to poly(methylmethacrylate) lens) and posterior capsular rupture during surgery lodds ratio 20.9, 95\% confidence intervals 2.3 to 187.9 ).

Conclusion: Silicone intraocular lens and rupture of the posterior capsule are risk factors of acute endophthalmitis after cataract surgery.
\end{abstract}

A cute infectious endophthalmitis after cataract extraction is a rare but potentially blinding complication. ${ }^{12}$ Although numerous studies have investigated possible risk factors for acute endophthalmitis, results have not been consistent..$^{3-9}$ Additionally, because the majority of these studies were conducted in the United States or Europe, it is unclear if they are applicable to other populations and settings. Therefore, we conducted a case-control study to examine risk factors of acute endophthalmitis after cataract extraction at an ophthalmic hospital in Singapore, a country with a multiethnic Asian population (77\% Chinese, 14\% Malay, $8 \%$ Indians, and $1 \%$ other ethnic groups).

\section{METHODS}

\section{Acute endophthalmitis cases and controls}

The study population was derived from patients who had extracapsular cataract extraction (ECCE) or phacoemulsification with or without intraocular lens (IOL) implantation performed at the Singapore National Eye Centre (SNEC) between I January 1996 and 31 December 2001. The institutional review board at the SNEC approved this study.

Postoperative endophthalmitis cases are prospectively identified at the SNEC by the clinical audit department. The diagnosis of acute endophthalmitis was made clinically, defined as patients presenting with symptoms of pain or worsening of visual acuity, with signs of hypopyon or vitreous clouding after cataract surgery. Management of endophthalmitis followed a standardised protocol at the SNEC, which typically entailed hospital admission, a review by a senior ophthalmologist (SPC), vitreous tap, antibiotic therapy, and vitrectomy according the Endophthalmitis Vitrectomy Study guidelines. ${ }^{10}$ Vitreous samples were sent for Gram stain microscopy and inoculated on blood, chocolate and Sabouroud's agar, and thioglycolate and brain-heart infusion broth to culture both anaerobic and aerobic microbial organisms at a central microbiological laboratory. Any growth of organisms was defined in this study as a case of culture positive endophthalmitis

We identified 34 cases of acute endophthalmitis presenting within 6 weeks after cataract surgery between 1996 and 2001. Of these, 21 were culture positive cases. Coagulase negative staphylococcus was the most common organism isolated $(n=12)$. Other organisms isolated included two cases of Staphylococcus aureus and alpha haemolytic streptococci, and single cases of Pseudomonas aeroginosa, Providencia sp, Enterobacter sp, group B streptococci, and Enterococcus sp.

For each case, we randomly selected three controls $(\mathrm{n}=102)$ from the cataract surgery list matched to the date of the operation of cases.

\section{Definition of risk factors}

A senior ophthalmic nurse who was masked to the study objectives and the case-control status reviewed all 136 clinical notes that were provided in a random order. The information was recorded on a standard form which did not include references to the diagnosis of endophthalmitis, culture results, or management (these data are entered prospectively by the clinical audit department). Information collected from the clinical notes include the following: patient demographics, medical and ocular history, type of anaesthesia, type and duration of operation, location and type of IOL, and intraoperative complications. IOL was categorised into three groups: poly(methylmethacrylate) (PMMA) lens (for example, 808A from Pharmacia), silicone lens (for example, SI$40 \mathrm{NB}$ from Allergen), and acrylic lens (for example, MA60BM from Alcon). One patient with a three piece PMMA lens implant was included in the PMMA group for analysis, while another patient who did not receive an IOL implant was excluded from this specific analysis. Posterior capsular rupture (PCR) was defined as present if there were any tears of the posterior capsule with or without vitreous loss noted during surgery.

\section{Statistical analysis}

We used logistic regression models to estimate the odds ratio (OR) and its 95\% confidence interval (CI) of endophthalmitis associated with a specific risk factor. Variables that were significant at the $p<0.10$ level in the univariate models were included in backward stepwise logistic regression models to select the final list of independent variables. All analyses were computed using SPSS (version 10.0, SPSS Inc, Chicago, IL, USA)

\section{RESULTS}

Cases and controls were similar in age (mean 66.8 (SD 12.9) years for cases and $66.4(10.4)$ years for controls, $p=0.86)$. Table 1 shows the distribution of possible risk factors in controls and endophthalmitis cases, and the results of univariate logistic regression analyses. Few variables were significantly associated with increased risk of endophthalmitis. 
Table 1 Univariate analysis of risk factors for acute endophthalmitis after cataract extraction

\begin{tabular}{|c|c|c|c|c|c|c|c|c|}
\hline \multirow[b]{2}{*}{ Risk factor } & \multirow[b]{2}{*}{ Definition } & \multirow{2}{*}{$\begin{array}{l}\text { Controls } \\
\text { No (\%) }\end{array}$} & \multicolumn{3}{|c|}{ All endophthalmitis } & \multicolumn{3}{|c|}{ Culture positive endophthalmitis } \\
\hline & & & No (\%) & OR $(95 \% \mathrm{CI})^{*}$ & p Value & No (\%) & OR $(95 \% \mathrm{CI})^{*}$ & p Value \\
\hline \multirow[t]{2}{*}{ Age } & $<65$ years & 34 (33.3) & $14(41.2)$ & 1.0 & 0.41 & $8(38.1)$ & 1.0 & 0.68 \\
\hline & 65 years or older & $68(66.7)$ & $20(58.8)$ & $0.7(0.3$ to 1.6$)$ & & 13 (61.9) & $0.8(0.3$ to 2.1$)$ & \\
\hline \multirow[t]{2}{*}{ Sex } & Men & $59(57.8)$ & $14(41.2)$ & 1.0 & 0.09 & 7 (33.3) & 1.0 & 0.04 \\
\hline & Women & $43(42.2)$ & $20(58.8)$ & 2.0 (0.9 to 4.3$)$ & & $14(66.7)$ & $2.7(1.0$ to 7.4$)$ & \\
\hline \multirow[t]{2}{*}{ Race } & Chinese & $86(84.3)$ & $25(73.5)$ & 1.0 & 0.16 & $17(81.0)$ & 1.0 & 0.70 \\
\hline & Non-Chinese & $16(15.7)$ & $9(26.5)$ & $1.9(0.8$ to 4.9$)$ & & $4(19.0)$ & $1.3(0.4$ to 4.3$)$ & \\
\hline \multirow{2}{*}{ Diabetes mellitus } & Absent & 77 (75.5) & $23(67.6)$ & 1.0 & 0.37 & $14(66.7)$ & 1.0 & 0.40 \\
\hline & Present & $25(24.5)$ & $11(32.4)$ & $1.5(0.6$ to 3.4$)$ & & $7(33.0)$ & $1.5(0.6$ to 4.2$)$ & \\
\hline \multirow{4}{*}{$\begin{array}{l}\text { Lid margin disease } \\
\text { (eg, blepharitis) } \\
\text { Preoperative antibiotics }\end{array}$} & Absent & $96(94.1)$ & $33(97.1)$ & 1.0 & 0.51 & $10(47.6)$ & 1.0 & 0.84 \\
\hline & Present & $6(5.9)$ & $1(2.9)$ & $0.5(0.1$ to 4.2$)$ & & $11(52.4)$ & $1.1(0.4$ to 2.8$)$ & \\
\hline & Yes & $83(81.4)$ & $32(94.1)$ & 1.0 & 0.09 & $19(90.5)$ & 1.0 & 0.32 \\
\hline & No & $19(18.6)$ & $7(5.9)$ & $0.3(0.1$ to 1.2$)$ & & $2(9.5)$ & $0.5(0.1$ to 2.1$)$ & \\
\hline \multirow[t]{2}{*}{ Eye } & Right & $51(50.0)$ & $17(50.0)$ & 1.0 & 0.99 & $10(47.6)$ & 1.0 & 0.84 \\
\hline & Left & $51(50.0)$ & $17(50.0)$ & $1.0(0.5$ to 2.2$)$ & & $11(52.4)$ & $1.1(0.4$ to 2.8$)$ & \\
\hline \multirow[t]{2}{*}{ Procedure } & ECCE & $47(46.1)$ & $10(29.4)$ & 1.0 & 0.09 & $4(19.0)$ & 1.0 & 0.03 \\
\hline & Phacoemulsification & $55(53.9)$ & $24(70.6)$ & 2.1 (0.8 to 4.7$)$ & & $17(81.0)$ & $3.6(1.1$ to 11.5$)$ & \\
\hline \multirow{2}{*}{ Duration of operation } & $<30$ minutes & $71(69.6)$ & $24(70.6)$ & 1.0 & 0.91 & $15(71.4)$ & 1.0 & 0.87 \\
\hline & 30 minutes or longer & $31(30.4)$ & $10(29.4)$ & $1.0(0.4$ to 2.2$)$ & & $6(28.6)$ & $0.9(0.3$ to 2.6$)$ & \\
\hline \multirow[t]{3}{*}{ Anaesthesia } & Local/regional & 93 (91.2) & $34(100.0)$ & - & - & $21(100.0)$ & - & - \\
\hline & Topical & $6(5.9)$ & - & & & - & & \\
\hline & General & $3(2.9)$ & - & & & - & & \\
\hline \multirow[t]{3}{*}{ IOL type $†$} & All PMMA & $51(50.5)$ & $15(45.5)$ & 1.0 & & $8(38.1)$ & 1.0 & \\
\hline & Silicone & $4(4.0)$ & $5(15.1)$ & $4.3(1.0$ to 17.8$)$ & 0.05 & $5(23.8)$ & $8.0(1.8$ to 36.1$)$ & 0.007 \\
\hline & Acrylic & $46(45.5)$ & $13(39.4)$ & $1.0(0.4$ to 2.2$)$ & 0.93 & $8(38.1)$ & $1.1(0.4$ to 3.2$)$ & 0.85 \\
\hline \multirow[t]{2}{*}{ IOL locationt } & Posterior chamber & $97(95.1)$ & $32(97.0)$ & 1.0 & 0.65 & $20(95.2)$ & 1.0 & 0.98 \\
\hline & Anterior chamber & $5(4.9)$ & $1(3.0)$ & $0.6(0.1$ to 5.4$)$ & & $1(4.8)$ & $1.0(0.1$ to 8.8$)$ & \\
\hline \multirow{2}{*}{ Posterior capsular rupture } & Absent & $101(99.0)$ & $29(85.3)$ & 1.0 & 0.01 & 17 (81.0) & 1.0 & 0.006 \\
\hline & Present & $1(1.0)$ & $5(14.7)$ & $17.4(2.0$ to 155.0$)$ & & $4(19.0)$ & 23.8 (2.5 to 225.6 ) & \\
\hline \multirow[t]{2}{*}{ Anterior vitrectomy } & Not performed & $101(99.0)$ & $30(88.2)$ & 1.0 & 0.02 & $18(85.7)$ & 1.0 & 0.02 \\
\hline & Performed & $1(1.0)$ & $4(11.8)$ & $13.5(1.5$ to 125.1$)$ & & $3(14.3)$ & $16.8(1.7$ to 171.0$)$ & \\
\hline \multirow{2}{*}{ Use of suture $\ddagger$} & No & $33(60.0)$ & 15 (62.5) & 1.0 & 0.83 & $12(70.6)$ & 1.0 & 0.43 \\
\hline & Yes & $22(40.0)$ & $9(37.5)$ & $0.9(0.3$ to 2.4$)$ & & $5(29.4)$ & $0.6(0.2$ to 2.0$)$ & \\
\hline
\end{tabular}

*Odds ratio (95\% confidence intervals) of endophthalmitis cases versus controls in association with a specific risk factor.

†No IOL implant for one case.

†Phacoemulsification cases only.

Endophthalmitis was significantly associated with use of silicone IOL when compared with PMMA IOL $(p=0.05)$, the occurrence of PCR during surgery $(p=0.01)$ or the need to perform anterior vitrectomy $(\mathrm{p}=0.02)$. A trend towards higher risk was seen for female sex, use of preoperative antibiotics, and phacoemulsification procedure $(\mathrm{p}<0.10)$. Results were largely similar in the analyses for culture positive endophthalmitis

Variables eligible for inclusion in the multivariable analyses were sex, preoperative antibiotics use, type of operation, IOL type, and PCR (all p<0.10 in the univariate analysis). Only IOL type and PCR were retained in the final multivariable models (table 2). The use of silicone IOL and the occurrence of PCR were independently associated with higher risk of endophthalmitis.

A supplementary analysis limiting to phacoemulsification cases only $(\mathrm{n}=79)$ showed similar results. Silicone IOL (OR $4.8,95 \%$ CI 1.1 to $20.5 .6, \mathrm{p}=0.03$, compared to PMMA and acrylic lens) and PCR (OR 19.2, 95\% CI 2.1 to 179.2, $\mathrm{p}=0.009$ ) were associated with an increased risk of endophthalmitis.

\section{DISCUSSION}

In this retrospective case-control study of Asian patients who had cataract surgery, we showed that silicone IOL (when compared with PMMA or acrylic IOL) and rupture of the posterior capsule were independently associated with acute endophthalmitis. This was seen in analysis confined to culture positive cases and phacoemulsification procedures only.

Silicone IOL is more hydrophobic than PMMA, and has therefore been hypothesised to be associated with a higher risk of bacterial contamination and subsequent endophthalmitis. Our results are compatible with experimental data ${ }^{11-13}$ and clinical studies $^{8}$ conducted in Western populations, although not all studies concur. ${ }^{14} 15$

PCR, a common complication of cataract surgery, was also a significant risk factor of endophthalmitis. This is supported

Table 2 Final multivariate models for acute endophthalmitis after cataract extraction

\begin{tabular}{|c|c|c|c|c|c|}
\hline \multirow[b]{2}{*}{ Risk factor } & \multirow[b]{2}{*}{ Definition } & \multicolumn{2}{|l|}{ All endophthalmitis } & \multicolumn{2}{|c|}{ Culture positive endophthalmitis } \\
\hline & & OR $(95 \% \mathrm{Cl})^{*}$ & p Value & OR $(95 \% \mathrm{Cl})^{*}$ & p Value \\
\hline \multirow[t]{4}{*}{ IOL type } & All PMMA & 1.0 & - & 1.0 & - \\
\hline & & 5.1 (1.2 to 21.6$)$ & 0.03 & $9.9(2.1$ to 46.3$)$ & 0.004 \\
\hline & Silicon & $0.9(0.4$ to 2.5$)$ & 0.88 & $1.0(0.3$ to 3.1$)$ & 0.97 \\
\hline & Acrylic & & & & \\
\hline \multirow[t]{2}{*}{ Posterior capsular rupture } & Absent & 1.0 & - & 1.0 & - \\
\hline & Present & 20.9 (2.3 to 187.9$)$ & 0.007 & 32.1 (3.3 to 313.0 ) & 0.003 \\
\hline
\end{tabular}

Odds ratio (95\% confidence intervals) of all endophthalmitis and culture positive endophthalmitis in backwards stepwise multiple logistic regression models that included the following covariates: IOL type, PCR, sex, and type of operation. Sex and type of operation dropped out of the final models. 
by some, ${ }^{236}$ but again not all, ${ }^{76}$ studies conducted elsewhere. The higher risk of endophthalmitis associated with PCR suggests that elimination of bacteria from the vitreous cavity may be less efficient than from the anterior chamber.

Limitations of this study should be mentioned. Firstly, information bias during data collection could have accentuated some associations and attenuated others. However, this was minimised by ensuring that the nursing officer who collected the data was masked to the study objectives and the case-control status. Secondly, because of the non-random allocation of patients, it is unclear whether selection biases might have influenced the results.

In summary, this study provides new data on the risk factors for acute endophthalmitis after cataract surgery in an Asian population. We found eyes with silicone IOL and rupture of the posterior capsule were more likely to develop acute endophthalmitis than eyes with PMMA or acrylic IOL and with intact posterior capsule. The findings here suggest that risk factors for acute endophthalmitis in Asians are largely similar to Western populations.

\section{ACKNOWLEDGEMENTS}

The authors would like to thank Dr Saw Seang Mei, Ms Eunice Loh, and Ms Jane Koh for their help with this paper.

\section{Authors' affiliations \\ TY Wong, S-P Chee, Singapore National Eye Center and Singapore Eye Research Institute, Singapore and Department of Ophthalmology, National University of Singapore \\ Correspondence to: Tien Yin Wong, Department of Ophthalmology, National University of Singapore, 10 Kent Ridge Crescent, Singapore} 119260, Singapore; ophwty@nus.edu.sg

Accepted for publication 28 May 2003

\section{REFERENCES}

1 Hughes DS, Hill RJ. Infectious endophthalmitis after cararact surgery. Br J Ophthalmol 1994;78:227-32.

2 Norregaard JC, Thoning $\mathrm{H}$, Bernth-Petersen $\mathrm{P}$, et al. Risk of endophthalmitis after cataract extraction: results from the International Cataract Surgery Outcomes study. Br J Ophthalmol 1997;81:102-6.

3 Javitt JC, Vitale S, Canner JK, et al. National outcomes of cataract extraction. Endophthalmitis following inpatient surgery. Arch Ophthalmol 1991;109:1085-9

4 Lertsumitkul S, Myers PC, O'Rourke MT, et al. Endophthalmitis in the western Sydney region: a case-control study. Clin Exp Ophthalmol 2001;29:400-5.

5 Puliafito CA, Baker AS, Haaf J, et al. Infectious endophthalmitis. Review of 36 cases. Ophthalmology 1982;89:921-9.

6 Menikoff JA, Speaker MG, Marmor M, et al. A case-control study of risk factors for postoperative endophthalmitis. Ophthalmology $1991 ; 98: 1761-8$.

7 Montan PG, Koranyi G, Setterquist HE, et al. Endophthalmitis after cataract surgery: risk factors relating to technique and events of the operation and patient history: a retrospective case-control study. Ophthalmology 1998; 105:2171-7.

8 Bainbridge JW Teimory $\mathrm{M}$, Tabandeh $\mathrm{H}$, et al. Intraocular lens implants and risk of endophthalmitis. Br J Ophthalmol 1998;82:1312-5.

9 Schmitz S, Dick HB, Krummenauer F, et al. Endophthalmitis in cataract surgery: results of a German survey. Ophthalmology 1999;106:1869-77.

10 Endophthalmitis Vitrectomy Study Group. Results of the endophthalmitis vitrectomy study. A randomised trial of immediate vitrectomy and of intravenous antibiotics for the treatment of postoperative bacterial endophthalmitis. Arch Ophthalmol 1995; 113:1479-96.

11 Cusumano A, Busin M, Spitznas M. Bacterial growth is significantly enhanced on foldable intraocular lenses. Arch Ophthalmol 1994;112:1015-16.

12 Garcia-Saenz MC, Arias-Puente A, Fresnadillo-Martinez MJ, et al. In vitro adhesion of Staphylococcus epidermidis to intraocular lenses. J Cataract Refract Surg 2000;26:1673-9.

13 Kodjikian L, Burillon C, Chanloy C, et al. In vivo study of bacterial adhesion to five types of intraocular lenses. Invest Ophthalmol Vis Sci 2002;43:3717-21.

$14 \mathrm{Ng} \mathrm{EW}$, Barrett GD, Bowman R. In vitro bacterial adherence to hydrogel and poly(methyl methacrylate) intraocular lenses. J Cataract Refract Surg 1996;22(Suppl 2):1331-5.

15 Schaversberger $J$, Amon $M$, Aichinger $D$, et al. Bacterial adhesion to rigid and foldable posterior chamber intraocular lenses: in vitro study. J Cataract Refract Surg 2003;29:361-6.

16 Blomquist PH, Rugwani RM. Visual outcomes after vitreous loss during cataract surgery performed by residents. J Cataract Refract Surg 2002;28:847-52. 